\title{
Qualitative and Quantitative Perfusion Parameters Determined by 3D Single-Shot GRASE ASL MR Imaging
}

\author{
Claus Kiefer ${ }^{*}$, Frauke Kellner-Weldon, Marwan El-Koussy, Martinus Hauf, Gerhard Schroth \\ Support Center for Advanced Neuroimaging, Institute of Diagnostic and Interventional Neuroradiology, \\ University of Bern (Inselspital), Bern, Switzerland \\ Email: $\left\{{ }^{*}\right.$ claus.kiefer, frauke.kellner-weldon, marwan.el-koussy, martinus.hauf, gerhard.schroth $\} @$ insel.ch
}

Received October 27, 2011; revised December 2, 2011; accepted December 13, 2011

\begin{abstract}
Rationale and Objectives: A particular arterial spin (ASL) labeling technique, called 3D-single-shot GRASE ASL is discussed with respect to the ability and limits of quantifying perfusion parameters. Materials and Methods: The technique enables the acquisition of perfusion weighted signal at multiple delay times (TI) in one scan. The readout part is a gradient and spin-echo combination (GRASE) that uses switched gradient rephrasing of signals to produce several times as many signals as turbo-spin-echo, which translates into faster imaging time and higher signal-to-noise ratio (SNR) per imaging time. The technique provides the possibility for model based quantification of cerebral blood flow and the determination of the bolus arrival information without use of contrast agent and thus the characterization and determination of regions that are supported by collaterals. Results: Whereas for a quantification of the permeability using ASL the SNR is not high enough, at least qualitative permeability maps can be determined, if an optimal homogenous SNR was guaranteed. This was accomplished in brain regions with a high blood supply, typically given in tumors, and by using a correction for coil sensitivity at the highest possible additional scaling. Conclusion: The single-shot 3D GRASE ASL can provide information about the principal blood supply, the transit delay of the blood flow due to a stenosis or collaterals and a qualitative measure of the permeability.
\end{abstract}

Keywords: ASL; Perfusion; GRASE; Model; Permeability

\section{Introduction}

Arterial spin-labeling (ASL) was introduced [1] as a noninvasive method capable of assessing cerebral perfusion and the temporal dynamics of arterial blood inflow.

Whereas real continuous labeling has drawbacks because it requires near continuous wave RF transmit capability (dual-coil) that is often not available on imagers or overloads the amplifier (duty cycle limits), the pulsed technique can be used on clinical scanners. In pulsed versions an instantaneous RF pulse (several milliseconds) is used to tag a slab of arterial blood. The implementation is straightforward and provides a tagging efficiency above $97 \%$ [2], which has been shown to be largely insensitive to variations in flow velocity. In comparison, the paper on pCASL, by [3], actually showed highly variable results (sometimes positive, sometimes negative) mostly due to incorrect setting of the phase. Computer simulations of pCASL predict an optimal tagging efficiency of $85 \%$ for arterial flow velocities in the limited range from 10 to $60 \mathrm{~cm} / \mathrm{s}$. It is theoretically and experimentally demonstrated in Wu's paper that the tagging efficiency

*Corresponding author. of pCASL is dependent technically upon the resonance offset, the flip angle of the RF pulse train, the phase and physiologically upon the blood flow velocity. The phase is a function of the slice selective gradient, the resonance offset of the tagging/control plane, and the flow velocity (a minor contribution compared to the former two). A large range in inversion efficiency measured across the subject group $(50 \%-76 \%)$ indicates that the velocity dependence of the amplitude modulated control efficiency may introduce additional variability into the perfusion calculations if not properly taken into account.

Swirling turbulent flow that causes multiple crossings and then return across the labeling plane could certainly be a problem. For measurement of cerebral perfusion, ASL of the blood flowing through the carotid- and vertebral arteries is typically performed at a axial plane, which is localized in the neck, just below the skull base. Turbulent blood due to arteriosclerosis or a stenosis of the vertebral or internal carotid artery at this level may signifycantly change the efficiency of the labeling. e.g., blood flow through a high grade stenosis is increased, resulting in a decrease of the labeling effect. The main pitfall in labeling blood inflow at the skull base and measuring the 
effect distally in the brain are collaterals. The transit time from the labeling pane to the brain is an essential parameter in determining the sensitivity of this method and for the quantification of perfusion. If the brain areas are supplied by collateral arteries from the opposite side or via branches of the external carotid artery, the perfusion may be sufficient, however, the transit time can be delayed resulting in an underestimation of the regional cerebral blood flow, if time between labeling and measurement is not long enough to detect also the delayed collateral inflow. The CASL signal change is predicted to be independent of the transit time if a sufficiently long post-labeling delay (PLD) is used $[4,5]$. The CBF values, measured by CASL are perfectly independent of transit time to the vascular compartment, for both gray and white matter as long as the transit time is shorter than the PLD. However, in case of a stenosis, this delay can be very long (in the range of more than two seconds) resulting in a signal loss due to T1 relaxation, which has to be compensated for by an increased number of acquisitions. In order to acquire full brain volumes, the acquisition time is in the range of 7 minutes for 120 volumes using pCASL. The quantification of CBF in brain areas, supplied by stenotic arteries and via collaterals however necessitates at least doubling the SNR (see Figure 1) and thus four times the number of acquisitions (in the example 480 acquisitions or 28 minutes).

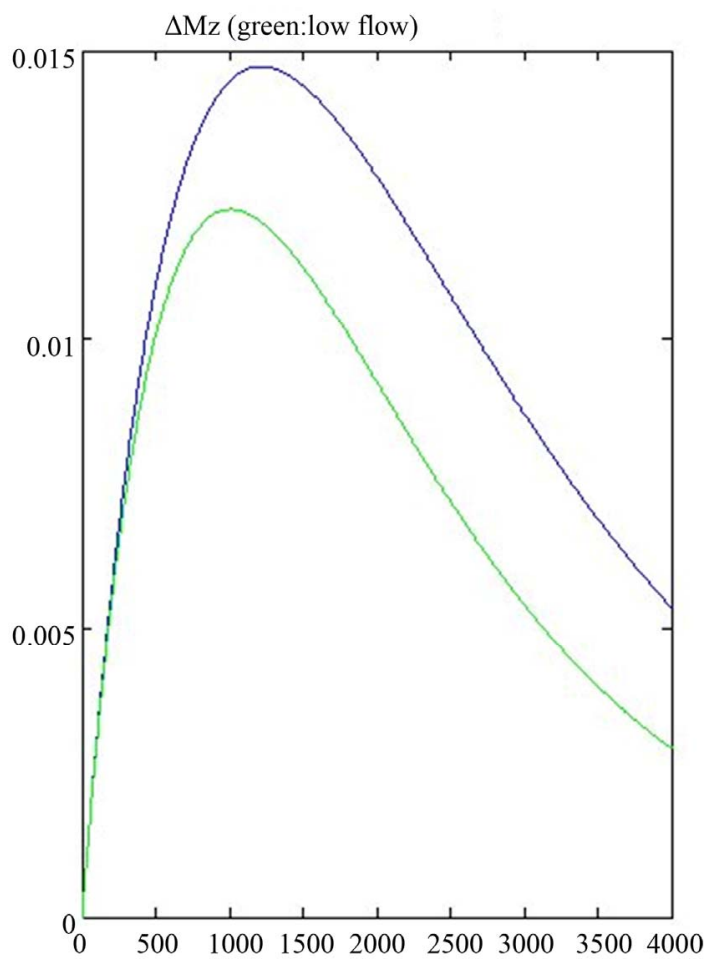

Figure 1. Effects of T1-relaxation on the ASL difference signal for high (black curve, $90 \mathrm{ml} / 100 \mathrm{~g} / \mathrm{min}$ ) and low flow (green curve, $30 \mathrm{ml} / 100 \mathrm{~g} / \mathrm{min}$ ) for time period of 4 seconds.
To overcome the problems with the labeling efficiency, the labeling slice and the transit times, a particular arterial spin labeling technique, called single-shot 3D-GRASE ASL, was recently introduced [6], that enables the acquisition of perfusion weighted signal at multiple delay times (TI) in one scan. One of the major issues in quantitative perfusion measurements using ASL, which might result in inaccurate perfusion values, is the contamination of the microvascular perfusion with different arrival times for the labeled blood in different regions. This problem can be solved by measuring the hemodynamic curve, acquired at several delay times, and evaluating it in the context of sophisticated perfusion models. Knowledge of the regional arrival times of arterial blood furthermore provide additional information to characterize the collateral flow and may potentially be used to identify hemodynamically impaired regions. The most common method to measure arrival times of blood is dynamic sampling of an injected bolus of contrast agent. However, due to the current concerns regarding contrast use in patients with poor renal function and ionizing radiation, an alternative without detrimental effects would be of great benefit.

In order to quantify the perfusion parameters a twocompartment model was used which is described in detail in reference [7].

In this work the GRASE ASL technique is discussed with respect to the ability and limits of quantifying perfusion parameters, especially the determination of the permeability of vessels.

\section{Materials and Methods}

GRASE (gradient and spin echo) is an imaging technique, that combines the essential features of turbo-spin echo (TSE) and echo-planar imaging (EPI) methods. It uses a train of refocusing $180^{\circ} \mathrm{RF}$ pulses as in TSE, placing additional gradient recalled echoes for each spin echo of the readout. GRASE therefore provides the option to influence the speed by setting a turbo factor as well as an EPI factor - so the gain in time as compared to conventional spin echo is the TSE turbo factor times the EPI factor. As a trade-off some image blurring and drop in SNR occur due to the longer multiple echo readout and modulations in signal intensity across $k$-space due to signal decay. Separately, both TSE and EPI experience these effects in subtly different ways. In EPI techniques, the strength of the successive gradient echoes can decay rapidly due to $\mathrm{T}_{2}^{*}$ effects, causing the later echoes to have a significantly reduced SNR as compared to the earlier echoes. However, in GRASE, the $180^{\circ} \mathrm{RF}$ pulses help to refocus intermittently the echoes to a maximum within the decay envelope. According to Guenther et al. the mean SNR of 3D-GRASE in gray matter therefore is $13.0 \pm 3.5$ and $4.2 \pm 0.9$ in white matter, for a $2 \mathrm{D}$-EPI 4.7 \pm 1.3 and $1.3 \pm 0.2$ respectively $(4.5 \mathrm{~mm}$ partition/slice 
thickness). The refocusing also helps to reduce the artifacts related to magnetic susceptibility heterogeneity that are so common in EPI imaging. But, in the case of fixed metallic hardware for example, GRASE will clearly have related artifacts that would be less obvious in similar TSE scans.

In GRASE, the combination of spin echoes and gradient echoes at differing times leads to modulation in measured signal strength over the multiple-echo readout time, and thus imposes signal modulations over $k$-space. Typically, the stronger spin echoes are used to fill the centre of $k$-space and the weaker gradient echoes are used in the periphery. This distribution tends to emphasize overall SNR with an image similar to TSE, at the expense of some fine detail as compared to a TSE scan with similar turbo factor. However, some authors have demonstrated that single shot GRASE can have improved spatial resolution when compared with single shot EPI. A large advantage of single-shot 3D techniques is that the whole image volume is acquired at the same inflow time TI. All partitions acquired throughout the echo train have the same amount of perfusion weighting since there is only one excitation pulse, which specifies the actual TI. Aliasing artifacts in the 3D-encoding direction are reduced tremendously after application of the modulated saturation pulses since the signal outside the imaging slab is nulled directly before readout.

The ASL preparation part, where the labeling takes places, is associated with problems concerning the suppression of stationary tissue and high inflow from great vessels. The latter could be accomplished by using small diffusion gradients, but the required strength of these gradients is difficult to estimate and time consuming to determine it during the examination, so we did not make use of it. Background suppression techniques [8,9] were suggested using multiple nonselective inversion pulses to null the signal of the stationary tissue while leaving the signal of the labeled blood untouched (for perfect pulses). Proposed technique uses modulated saturation pulses, which result in two saturation bands on both sides of the imaging slab. This suppresses intravascular blood flowing into the imaging slab from not only one but also from both sides, thus also reducing the signal of most venous vessels.

Because GRASE-ASL is based on the FAIR labeling scheme [10], there are no problems with magnetization transfer effects. This labeling method is based on the subtraction of images acquired alternately using a sliceselective and a non-selective inversion recovery sequence, as opposed to techniques, in which blood is labeled only proximally to the measured slice in the labeling phase, and not in the control phase of the sequence. The magnetization transfer (MT) artifacts may then occur in the perfusion-weighted images arising from macromolecular spins in the imaged slice that have been excited by the labeling radiofrequency (RF) pulse.

RF pulses or pulse trains used for tagging have imperfect slice profiles and can contaminate the magnetization in the ROI. These effects can be on the order of a few percent of the static tissue magnetization, which is the same order as the ASL signal. Pulsed ASL techniques typically use hyperbolic secant (sech) or similar adiabatic pulses for efficient inversion that is relatively insensitive to both B1 inhomogeneity and resonance offsets. Modified sech pulses such as frequency-offset-corrected inversion (FOCI) pulses were used in the sequence according to the proposals of Guenther et al. [6] For an optimized parameter set the FOCI pulses were calculated and the inversion profile of the longitudinal magnetization was simulated in Matlab using the Bloch equations.

Perfusion parameters $\mathrm{CBF}$ and permeability surface area product (PS) were estimated by fitting a FAIR model without backflow [7] to the GRASE ASL difference data series. This model corrects for the assumption that the capillary wall has infinite permeability to water. The model incorporates an extravascular and a blood compartment with the permeability surface area product (PS) of the capillary wall characterizing the passage of water between the compartments. The model predicts that labeled spins spend longer in the blood compartment before exchange. Permeability of the capillary wall to water is measured in terms of PS, the permeability (P) surface area (S) product of brain capillaries to water, per volume of tissue. It has been measured by a number of different methods in a number of species. Published values in whole human brain vary from $0.9-1.7 \mathrm{~min}^{-1}$ with a mean value of $1.2 \mathrm{~min}^{-1}$ [11]. A blood water compartment and an extravascular water compartment, each with corresponding volumes and longitudinal relaxation times, are separated by semi-permeable endothelium. An extra component was introduced to the differential equations of the model, which accounts for labeled water crossing between the two compartments through the permeable capillary wall. Like $T_{1}$, the $T_{2}^{*}$ values for blood and extravascular water are also different. The difference signal will change as water moves between the two compartments. $R 1=1 / T_{1}$, which is necessary for the model, was estimated from the same ASL dataset. The BAT-map, delivered by 3D-single-shot GRASE, was used as a measure for the transit time in order to correct for a distribution of transit times centered on MTT. The PS parameter maps finally were qualitatively compared with the apparent k21 parameter maps of the DSC based evaluation using the Nordic software [12] which includes the leakage correction algorithm of reference [13].

The sequence was delivered with a special feature, an option called additional scaling that allows to influence the image intensity and SNR in addition to the scaling 
done by the software of the manufacturer. This option in some cases had to be set up to $70 \%$ to set the image intensity within the brain matter to values near 3500 (total range is 0.4095).

The GRASE-ASL was applied to patients with a tentorium-meningeoma and a high grade stenosis (ICA left). GRASE and DSC sequences were performed on a 3 Tesla scanner, 32-channel receiver coil, additional scaling 50\% (maximum intensity value 3500 ). Postprocessing of GRASE was done with a MATLAB written software, the DSC data was evaluated with Nordic-ICE software (options leakage correction and maps). The sequence parameters for the GRASE are as follows: voxel size: $4.7 \times 4.7 \times 4.0 \mathrm{~mm}$, matrix $64 \times 64$, TR $3200 \mathrm{~ms}$, TE $12.8 \mathrm{~ms}$, Averages 2, Slice partial Fourier 6/8, PAT mode None, Sat. region Thickness $120 \mathrm{~mm}$, Phase oversampling $6 \%$, Slice oversampling $15.4 \%$, Slices per slab 26, FoV read $300 \mathrm{~mm}$, FoV phase 50.0\%, Slice thickness $4.0 \mathrm{~mm}$, Dimension 3D, Reordering Centric, Bandwidth $2790 \mathrm{~Hz} / \mathrm{Px}$, Echo spacing $0.5 \mathrm{~ms}$, Turbo factor 23, EPI factor 17, Bolus length $4000 \mathrm{~ms}$. The bandwidth was determined by minimizing the artifacts in the difference images measuring a stationary spherical water phantom. The sequence currently just allows to select $50 \%$ or $68 \%$ FoV phase which is related to 6 or 9 minutes measurement time. With regard to the shorter time we choose $50 \%$ and accepted the infolding artifacts.

For the background suppression scheme we used $\mathrm{T}$ lopt $=700 \mathrm{~ms}$ and delay $=100 \mathrm{~ms}(\mathrm{TI}=$ inflow time delay, inflow time $=$ start $+($ len -1$) \times$ inc $=2800 \mathrm{~ms}($ start $=200$, inc $=200$, len $=14)$ ). The two inversion pulses then are at times $\tau 1=1,265,860 \mu \mathrm{s}, \tau 2=2,363,490 \mu \mathrm{s}$.

For the saturation mode a series of sinc pulses with a flip angle 90 degree, duration $\mathrm{T}=10240 \mu$ s, was used the saturation was performed before and after labeling.

The parameter for the FOCI inversion rf pulse shapes (beta, mu, FOCI-factor (flat top value of the gradient modulation), flip, phase correction factor (slab shift resp. offcenter frequency shift used to determine the rf-frequency) were: $900,24,1.0,500,10.000$.

\section{Results}

The problem of a lack of SNR and T1-relaxtion is illustrated in a simulation of perfusion using flow modified Bloch equations: $\mathrm{T} 1$ for tissue and labeled blood at $3 \mathrm{~T}$ were set to $1200 \mathrm{~ms}$ (gray matter) and $1600 \mathrm{~ms}$ respectively (Figure 1). The maximal difference signal (control-label) with respect to the normalized initial signal is only 0.015 (1.5\% of the entire signal per voxel) and is further reduced to 0.005 for high flow $(90 \mathrm{ml} / 100 \mathrm{~g} / \mathrm{min})$ and 0.003 for low flow $(30 \mathrm{ml} / 100 \mathrm{~g} / \mathrm{min})$ after a delay of $4 \mathrm{sec}$. Especially the last value for low flow characterizes the problem associated with the ASL based quantification of $\mathrm{CBF}$ with respect to the SNR within regions with a pathological reduction of flow, as in ischemic or stenotic tissue areas.

The gain in speed in our GRASE sequence is roughly

$$
\left(\frac{1}{\mathrm{TSE}-\text { turbofactor }}\right) \times\left(\frac{1}{\text { EPI }- \text { factor }}\right)=\frac{1}{23} \times \frac{1}{17}=\frac{1}{391}
$$

which allows to acquire the $\mathrm{N}=14$ volumes (26 slices) in a time of 6 minutes. The 3D-infolding artifacts only concern the upper two slices whereas the right-left infolding due to the small FOV phase $(50 \%)$ was a problem in about four of forty cases. The FOCI-pulses and the inversion profile for a slab shift of $100 \mathrm{~mm}$, shown in Figure 2, indicate a nearly perfect rectangular shape. The fitting results in Figure $\mathbf{3}$ demonstrate that the hemodynamic behavior can be reproduced by the two-compartment perfusion model - the correlation of measured and fitted curves is better than 0.95. The related CBF and BAT maps for the meningeoma patient are shown in Figures 4.

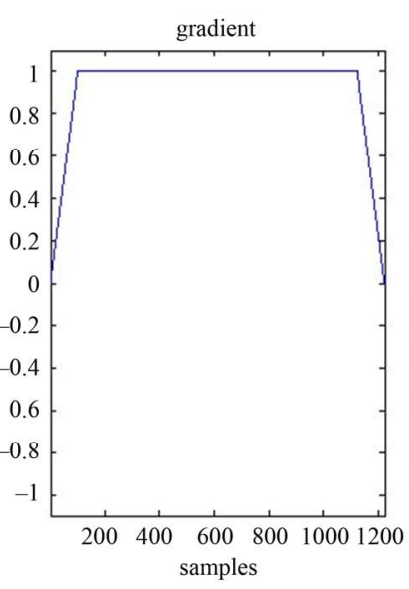

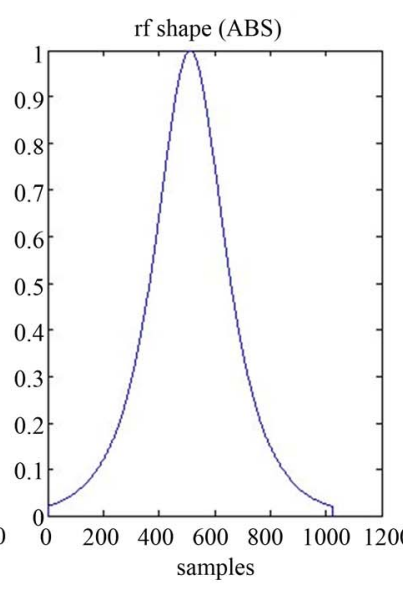

(a)
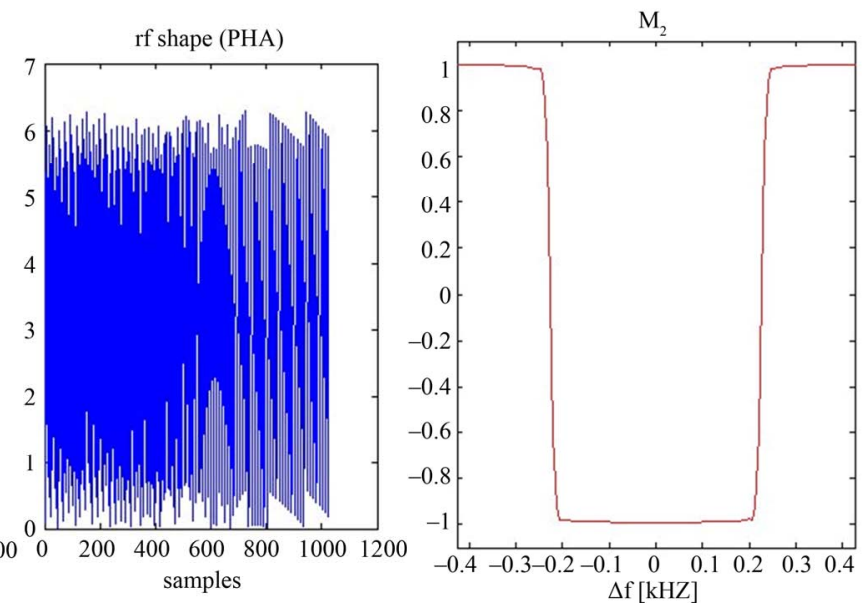

(b)

Figure 2. Off-resonance FOCI-pulse for a slab shift of $100 \mathrm{~mm}$ (a) gradient, rf-amplitude, rf-phase; and (b) inversion profile. 

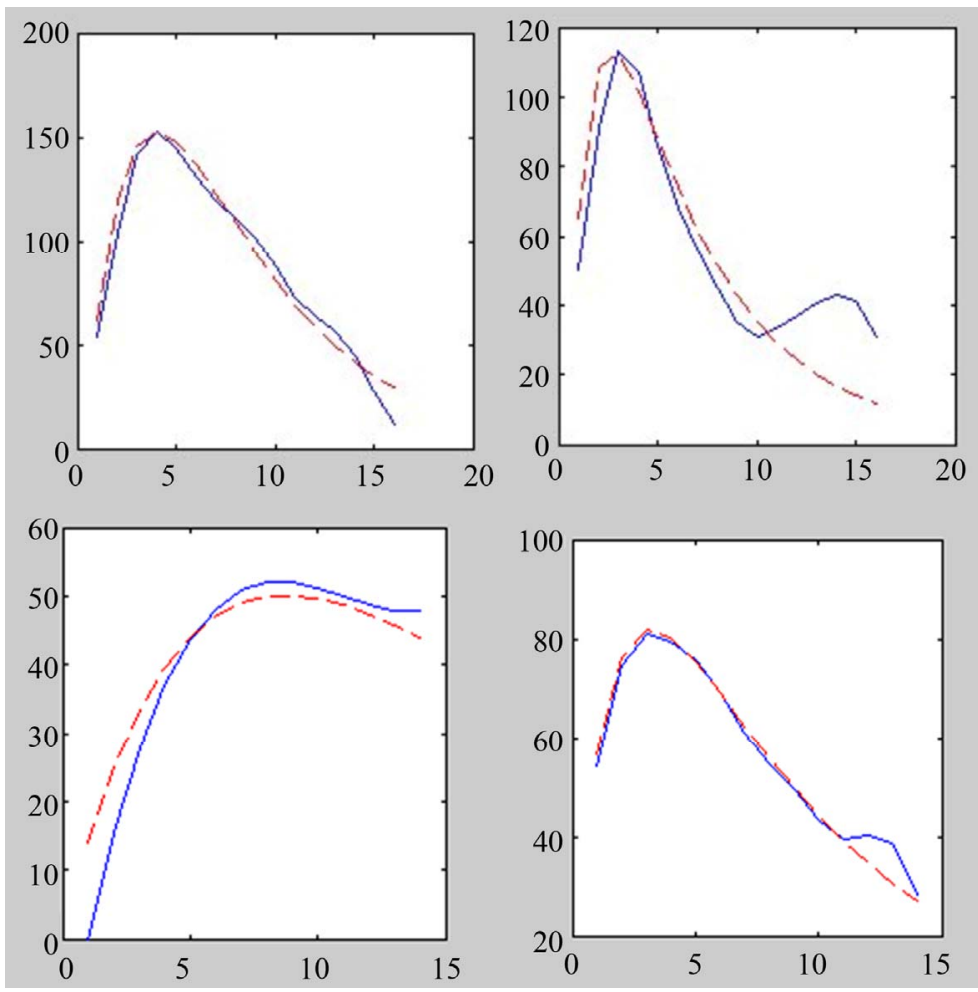

Figure 3. For four different representative voxels the model based calculated curves (red dashed curves) are compared to the measured curves (blue solid curves): the correlation of the measured and fitted curves is better than 0.95 .

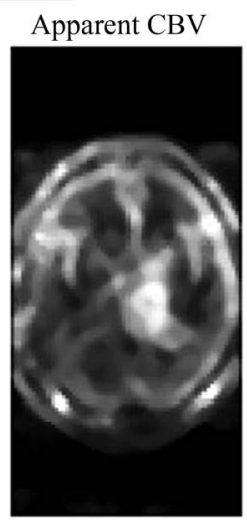

Anatomie

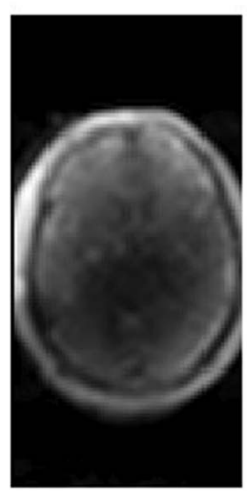

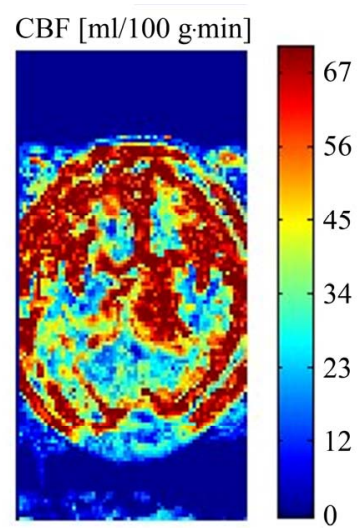

Bolus arrival time $[\mathrm{ms}]$

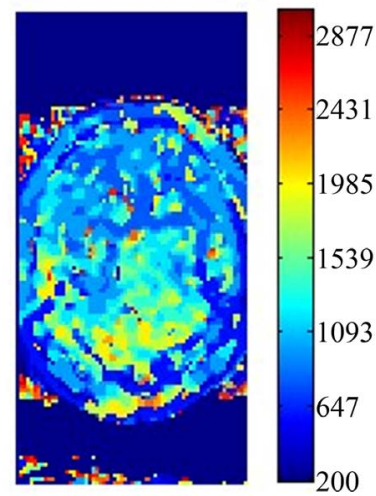

Figure 4. Integrated GRASE inflow curves (apparent CBV), CBF, anatomy, BAT maps for a patient with a meningeoma (before embolization). 
The $\mathrm{CBF}$ values in white matter vary between 10 and 20 $\mathrm{ml} / 100 \mathrm{~g} / \mathrm{min}$ which is in good agreement with the work of Pohmann et al. [14], where $15.6 \pm 3.2 \mathrm{ml} / 100 \mathrm{~g} / \mathrm{min}$ in the left and $15.2 \pm 4.8 \mathrm{ml} / 100 \mathrm{~g} / \mathrm{min}$ in the right hemispheric white matter were reported. In Figure 5 the difference images are shown for TI ranging from $200 \mathrm{~ms}$ to $2800 \mathrm{~ms}$ in steps of $200 \mathrm{~ms}$. Due to a high blood supply of the meningeoma, and thus a high contribution of the CBF related signal to the measured signal, the PS parameter derived from the GRASE data is at least qualitatively comparable to the apparent leakage (K21) parameter of the DSC evaluation (Figure 6).

It is furthermore interesting to note that the bolus length doesn't seem to have a great influence on the CBF values in gray matter: for bolus lengths $b=2000,3000$ and $4000 \mathrm{~ms}$ the (mean/maximum) $\mathrm{CBF}$ values in the cortex were (56/101), (64/104) and (61/103) $\mathrm{ml} / 100 \mathrm{~g} /$ min respectively. This was the reason why we did not limit the bolus length by saturation pulses but used the full length of 4 seconds in order to achieve the maximum SNR for pulsed ASL. In the case of the patient with the left ICA occlusion (Figure 7) the apparent CBV, the $\mathrm{CBF}$ and BAT maps are in excellent agreement with the indication and the expectations. In a few voxels within areas on the stenotic brain hemisphere and the white matter the $\mathrm{CBF}$ values are underestimated $(<10 \mathrm{ml} / 100$ $\mathrm{g} / \mathrm{min}$ ) due to a too low SNR. The power of the multiple delay technique however detecting collaterals is demon- strated in Figure 8: within the stenotic brain hemisphere the supply by a vessel with delayed blood arrival but with a relatively high CBF value between 50 and $60 \mathrm{ml} /$ $100 \mathrm{~g} / \mathrm{min}$ is presented.

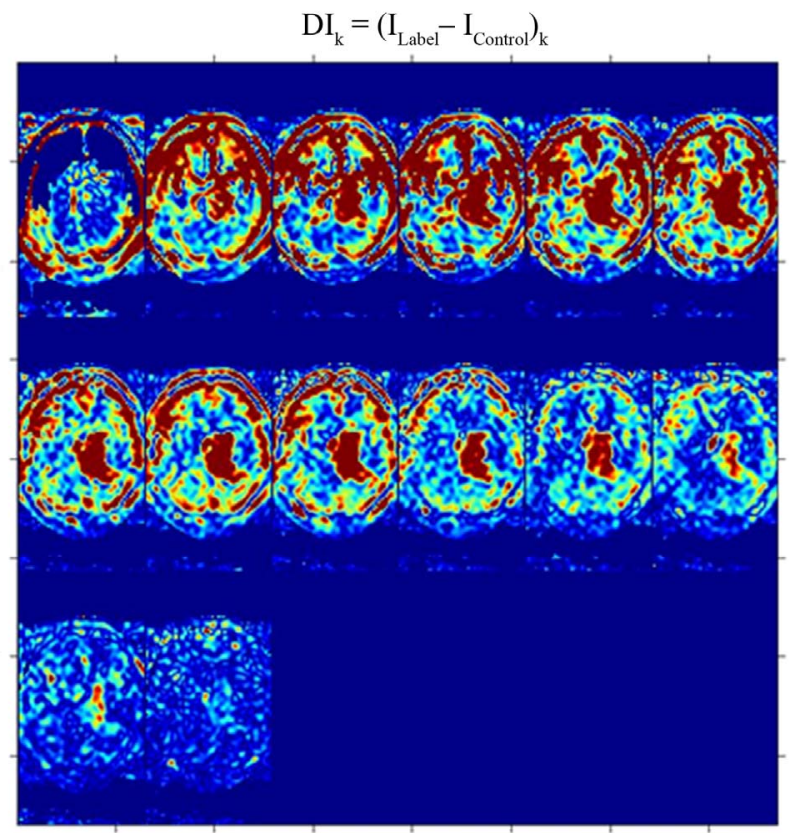

Figure 5. Dynamic difference maps (one slice, $\mathrm{TI}=\mathbf{2 0 0}$, $400, \ldots, 2800 \mathrm{~ms}$ ) for the patient with the meningeoma (before embolization).

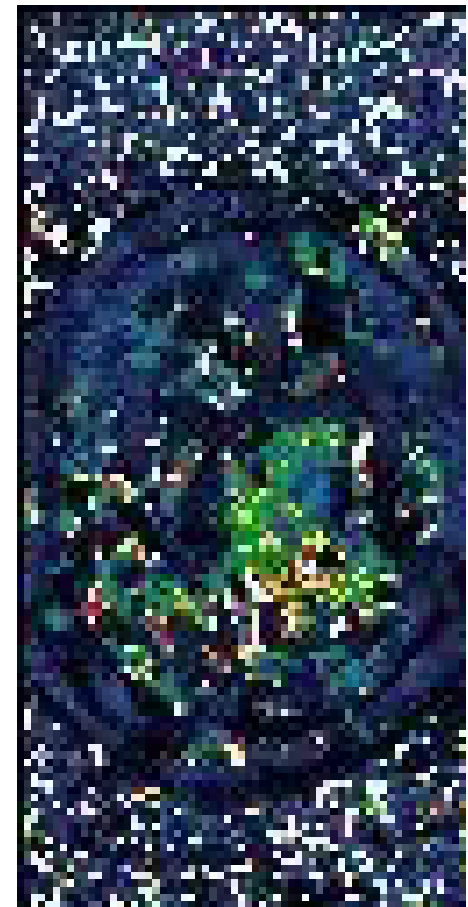

(a)

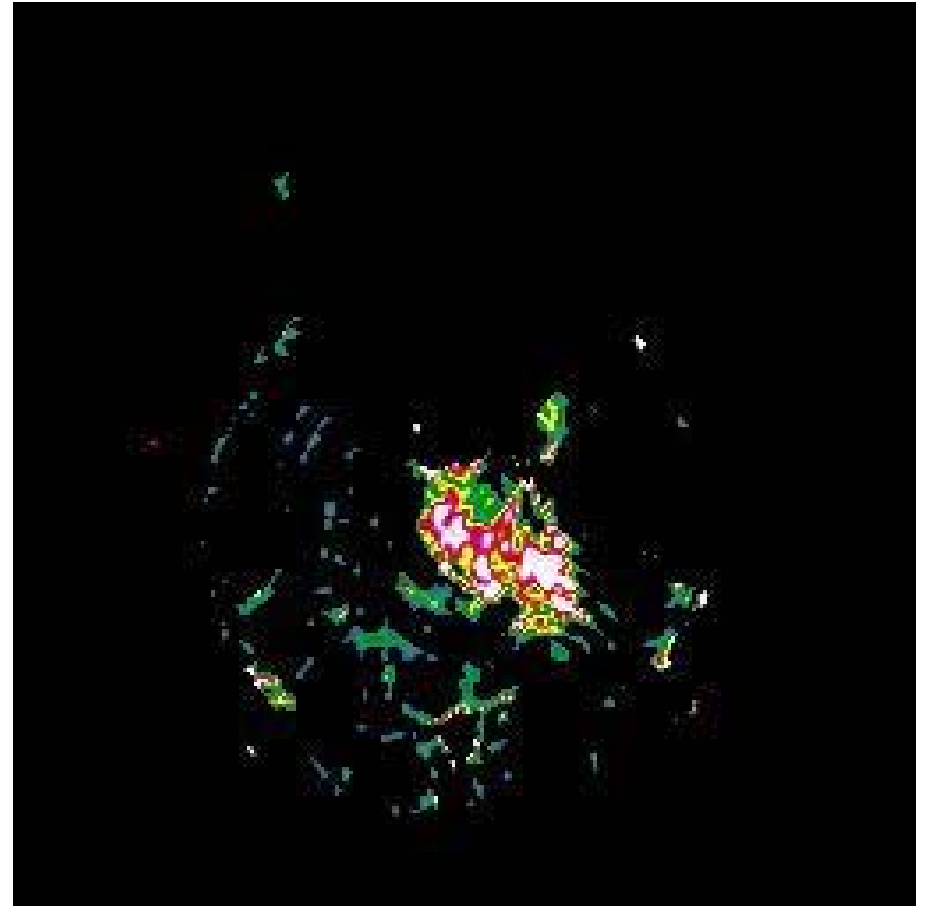

(b)

Figure 6. Permeability (a) and k21 (b) maps calculated from GRASE-ASL and Nordic (DCE) data sets resp. for the patient with the meningeoma (before embolization). 


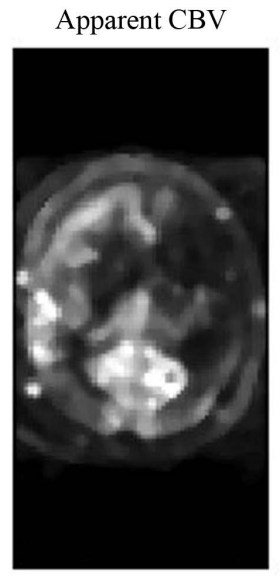

Anatomie

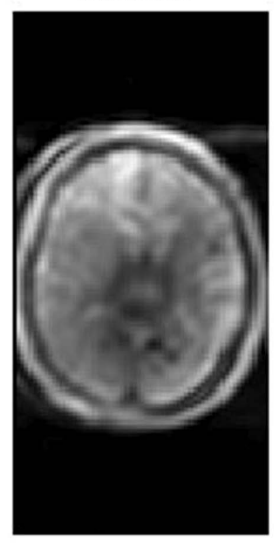

CBF $[\mathrm{ml} / 100 \mathrm{~g} \cdot \mathrm{min}]$

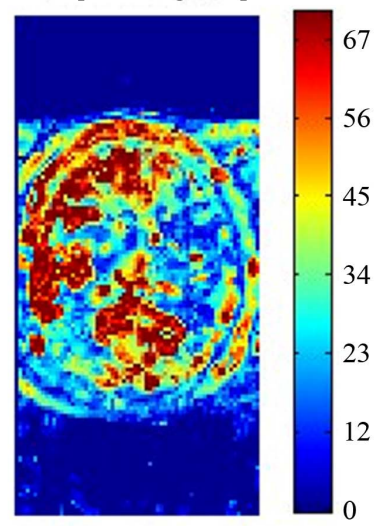

Bolus arrival time [ms]

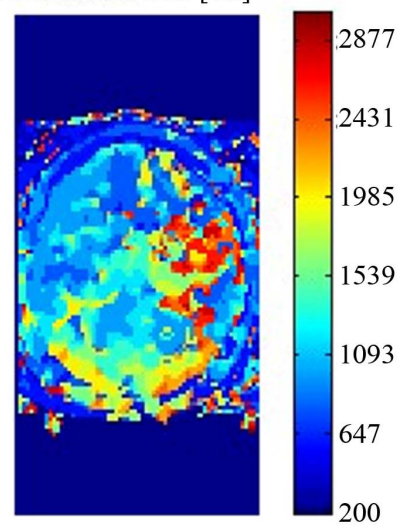

Figure 7. Patient with a stenosis (left ICA occlusion): integrated inflow (apparent CBV), CBF, anatomy and bolus arrival.
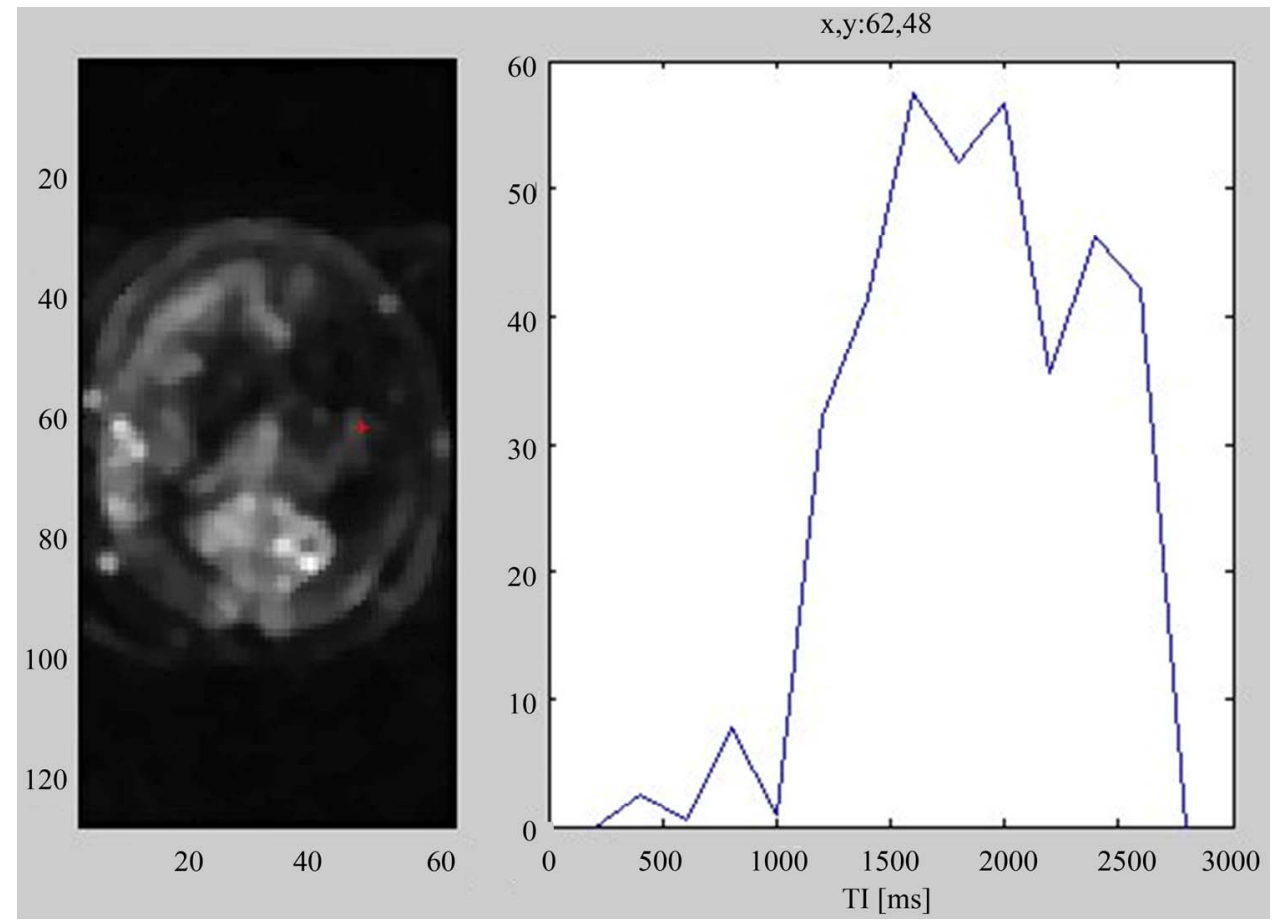

Figure 8. Patient with the stenosis (see Figure 7): difference signal picked up at the marked position (peak value is equal to the CBF value). 


\section{Discussion}

The 3D-Single-Shot GRASE ASL technique provides the possibility for model based quantification of perfusion and the determination of the bolus arrival information without use of contrast agent. In order to get at least qualitative leakage (K21) or permeability-surface (PS)maps, an optimal homogenous SNR should be guaranteed. This is accomplished in brain regions with a high blood supply, typically given in tumors, and by using a correction for coil sensitivity at the highest possible additional scaling. Whereas for a quantification of the permeability using ASL the SNR is not high enough, as could be demonstrated in [15], at least qualitative permeability maps can be determined, if an optimal homogenous SNR was guaranteed. In summary the single shot 3D-GRASE ASL technique is a promising perfusion imaging application for clinical routine. The optimized FOCI pulses for on/off-resonance inversion guarantee a non-disturbed magnetization behavior within the regions of interest and a labeling efficiency of $97 \%$ which is clearly higher than the one of pcasl $(\leq 85 \%$, velocity and turbulence dependent). The effects of swirling turbulences and high flow in the labeling slice on the labeling efficiency are avoided. The model based evaluation of hemodynamic enables to determine the bolus arrival resp. transit time parameter and thus the characterization and determination of regions that are supported by collaterals. The model based evaluation of hemodynamic also enables to derive qualitative permeability and leakage maps so that at least ratios of pathological and normal tissue can be determined.

In order to investigate the effects of different transit times on the ASL signal a sufficient SNR must be guaranteed, especially in case of stenosis. This however necessitates, as already mentioned, measurement times in the range of 30 minutes and more, the cooperation of the patient - both conditions can currently not easily be met by the patients. Therefore, a direct comparison of pCASL and GRASE especially with respect to the quantification of the CBF in stenotic and collateral supplied regions is projected. Future experiments will be performed on perfusion phantoms for this reason, enabling long measurement times and artificial stenoses.

In view of the current parameterization of the GRASE sequence (TI $=0-2800 \mathrm{~ms}$ ) it becomes clear that the essential contributions to the perfusion signal are dominated by the arteries and arterioles. Measurements of the late parenchyma phase $(\max . \mathrm{TI}=4-5 \mathrm{sec})$ however would implicate long measurement times as well, which is not acceptable for the clinical routine and the patientsin case of three time points $(\mathrm{TI}=3500,4000,4500 \mathrm{~ms})$ and $\mathrm{N}=16$ averages (necessary at 3Tesla) an additional measurement time of 16 minutes has to be taken into account. Due to the limitations related to the maximum duty cycle of the rf amplifier, the measurement time, the T1-relaxation and the SNR, the ASL technique currently only depicts restricted clinical insights into perfusion processes.

Beside these aspects there are some problems related to a right-left infolding of big vessels and/or the cranium. This depends on the limitation of the FOV (and matrix size) in the R-L direction (phase) to $50 \%$ and the fact that this parameter is not arbitrarily adjustable - with respect to the EPI factor (maximum $16-17$ ) the next value is $62 \%$ - $68 \%$ which corresponds to 9 - 10 minutes instead of 6 minutes measurement time.

Concerning the calculation of the images and additional sequence features, a special option for coil sensitivity correction (prescan-normalize) had to be implemented in the sequence program code to ensure a homogeneous SNR over the entire slice. It is also currently being checked, if the post-processing steps for the additional scaling and the prescan-normalize (up to now in this order) can be exchanged to maximize the SNR and its homogeneity.

In face of all these facts however, the single-shot 3D GRASE ASL can provide information about the principal blood supply, the transit delay of the blood flow due to a stenosis or collaterals and a qualitative measure of the permeability.

\section{Acknowledgements}

This article was financially supported by the Swiss National Science Foundation: SPUM Consortium (33CM30124114). We also are grateful to Matthias Guenther for useful discussions.

\section{REFERENCES}

[1] J. A. Detre, W. G. Zhang, D. A. Roberts, A. C. Silva, D. S. Williams, D. J. Grandis, A. P. Koretsky and J. S. Leigh, "Tissue Specific Perfusion Imaging Using Arterial Spin Labeling," NMR in Biomedicine, Vol. 7, No. 1-2, 1994, pp. 75-82. doi:10.1002/nbm.1940070112

[2] E. C. Wong, R. B. Buxton and L. R. Frank, "A Theoretical and Experimental Comparison of Continuous and Pulsed Arterial Spin Labeling Techniques for Quantitative Perfusion Imaging," Magnetic Resonance in Medicine, Vol. 40, No. 3, 1998, pp. 348-355. doi: $10.1002 / \mathrm{mrm} .1910400303$

[3] W. C. Wu, M. Fernandez-Seara, J. A. Detre, et al. "A Theoretical and Experimental Investigation of the Tagging Efficiency of Pseudocontinuous Arterial Spin Labeling," Magnetic Resonance in Medicine, Vol. 58, No. 5, 2007, pp. 1020-1027. doi:10.1002/mrm.21403

[4] T. Mildner, H. E. Moller, W. Driesel, et al. "Continuous Arterial Spin Labeling at the Human Common Carotid Artery: the Influence of Transit Times," NMR in Biomedicine, Vol. 18, No. 1, 2005, pp. 19-23.

doi: $10.1002 /$ nbm. 917 
[5] D. C. Alsop and J. A. Detre, "Reduced Transit Time Sensitivity in Noninvasive Magnetic Resonance Imaging of Human Cerebral Blood Flow," Journal of Cerebral Blood Flow \& Metabolism, Vol. 16, No. 6, 1996, pp. 1236-1249. doi:10.1097/00004647-199611000-00019

[6] M. Guenther, K. Oshio and D. A. Feinberg, "Single-Shot 3D Imaging Techniques Improve Arterial Spin Labeling Perfusion Measurements," Magnetic Resonance in Medicine, Vol. 54, No. 2, 2005, pp. 491-498. doi: $10.1002 / \mathrm{mrm} .20580$

[7] L. M. Parkes and P. S. Tofts, "Improved Accuracy of Human Cerebral Blood Perfusion Measurements Using Arterial Spin Labeling: Accounting for Capillary Water Permeability," Magnetic Resonance in Medicine, Vol. 48, No. 1, 2005, pp. 27-41. doi:10.1002/mrm.10180

[8] F. Q. Ye, J. A. Frank, D. R. Weinberger, et al., "Noise Reduction in 3D Perfusion Imaging by Attenuating the Static Signal in Arterial Spin Tagging (ASSIST)," Magnetic Resonance in Medicine, Vol. 44, No. 1, 2000, pp. 92-100.

doi:10.1002/1522-2594(200007)44:1<92::AID-MRM14> 3.0.CO;2-M

[9] S. Mani, J. Pauly, S. Conolly, et al., "Background Suppression with Multiple Inversion Recovery Nulling: Applications to Projective Angiography," Magnetic Resonance in Medicine, Vol. 37, No. 6, 1997, pp. 898-905. doi:10.1002/mrm.1910370615
[10] S. G. Kim, "Quantification of Relative Cerebral Blood Flow Change by Flow-Sensitive Alternating Inversion Recovery (FAIR) Technique: Application to Functionalmapping," Magnetic Resonance in Medicine, Vol. 34, No. 3, 1995, pp. 293-301. doi:10.1002/mrm.1910340303

[11] O. B. Paulson, M. M. Hertz, T. G. Bolwig, et al., "Filtration and Diffusion of Water across the Blood-Brain Barrier in Man," Microvascular Research, Vol. 13, No. 1, 1997, pp. 113-124. doi:10.1016/0026-2862(77)90120-0

[12] Nordic Software. http://www.nordicsoftware.com/

[13] J. L. oxerman, K. M. Schmainda and R. M. Weisskoff, "Relative Cerebral Blood Volume Maps Corrected for Contrast Agent Extravasation Significantly Correlate with Glioma Tumor Grade, Whereas Uncorrected Maps Do Not," American Journal of Neuroradiology, Vol. 27, No. 4, 2006, pp. 859-867.

[14] R. Pohmann, "Accurate, Localized Quantification of White Matter Perfusion with Single-Voxel ASL," Magnetic Resonance in Medicine, Vol. 64, No. 4, 2010, pp. 1109-1113. doi:10.1016/0026-2862(77)90120-0

[15] J. P. Carr, D. L. Buckley, J. Tessier, et al., "What Levels of Precision Are Achievable for Quantification of Perfusion and Capillary Permeability Surface Area Product Using ASL?" Magnetic Resonance in Medicine, Vol. 58, No. 2, 2007, pp. 281-289. doi:10.1002/mrm.21317 